\title{
Color Fidelity in Computer Graphics: a Survey
}

\author{
Gilles Rougeron and Bernard Péroche \\ Ecole des Mines de Saint Etienne \\ LISSE \\ 158 Cours Fauriel \\ 42023 Saint Etienne Cedex 2, France \\ email : rougeron@emse.fr peroche@emse.fr
}

\begin{abstract}
The purpose of this paper is to make a state of the art for color fidelity in computer graphics. Color fidelity includes three steps. The first one is the spectral rendering phase which attributes a spectrum to each pixel of a picture. During the second step, a spectral data is transformed into a set of tristimulus values in the $X Y Z$ color space. The purpose of the third step, called Color Reproduction Function, is to determine the RGB values displayable on the screen, in such a way that subjective fidelity is reached. We especially detail the two last steps of the color fidelity process; we also point out the work still remaining to be done in this field and we propose some research ways.
\end{abstract}

Keywords: Spectral Rendering; Colorimetry; Vision Model; Color Monitor Model.

\section{Introduction}

An important topic in computer graphics is realistic image synthesis, whose main goal is to capture the visual appearance of modeled scenes. This topic may be decomposed into two sub-themes. The first one is concerned with physically-based rendering methods which enable to simulate the propagation of light energy in scenes. The second sub-theme is linked to the problem of visualizing the computation done during the previous simulation step.

This survey will only deal with the topic of visualization, which seems to have been less studied than physically-based rendering methods. The theoretical goal of this visualization step is to create for the screen observer the same sensations as if he were placed in the real scene. Actually, these sensations are merely suggested, which means that an objective fidelity is replaced by a subjective one.

Let us consider Figure 1. The visualization step is outlined on the right part of the figure, and the color fidelity problem lies in the comparison between the left and the right part of the diagram.

As may be seen on the right part of Figure 1, the

(C) The Eurographics Association 2002. Published by Blackwell Publishers, 108 Cowley Road, Oxford OX4 1JF, UK and 238 Main Street, Cambridge, MA 02142, USA. changeover from a modeled world to a picture displayed on a screen requires several steps.

The first one, briefly described in section 2 , is the spectral rendering phase, which permits to associate a spectrum with each pixel of the picture. The second step, developed in section 3, deals with the transformation of spectral data into a set of tristimulus values in the CIE trichromatic $X Y Z$ color space. The purpose of the third step, called Color Function Reproduction, is to determine the $R G B$ values displayable on the screen, in such a way that subjective fidelity can be reached. This step requires the description of a vision model, contained in section 4 , and the assessment of the possibilities of a color monitor, made in section 5. The determination of this Color Function Reproduction, although very important, certainly is one of the problems addressed in this paper among the less studied in the literature.

\section{The spectral rendering step}

As the goal of physically-based rendering is to simulate the behaviour of light in scenes, light sources and the interaction of light with materials have to be modeled $^{1}$. 


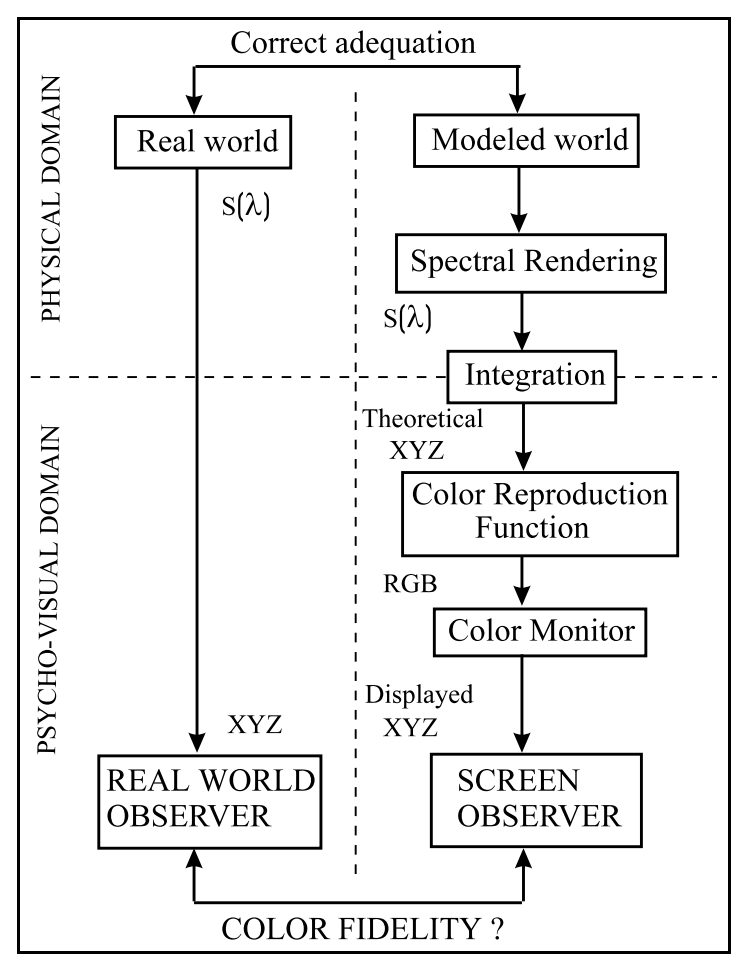

Figure 1: Visualization in image synthesis

Modeling a light source requires the knowledge of three physical characteristics: The geometry of the source, its luminous intensity, which can be described by goniometric diagrams, and its spectral power distribution which is defined by an emission spectrum in the visible wavelength domain.

During the last years, a great deal of work was concerned with global illumination models, which allow to simulate the interaction between light and materials. It would be too long to develop these models here: For more explanations, the reader may refer to ${ }^{2},{ }^{3}, 4$ or ${ }^{5}$.

We only want to emphasize the fact that for the reflection of light onto a surface, the right notion is the Bidirectional Reflectance Distribution Function (BRDF) $\rho(\lambda)$. It relates the reflected radiance at a point $M$ of the surface of a material to the incident radiance at this same point, by the formula:

$L_{\lambda}\left(M, \overrightarrow{\omega_{r}}\right)=\int_{\Omega_{i}} \rho_{\lambda}\left(M, \overrightarrow{\omega_{r}}, \overrightarrow{\omega_{i}}\right) L\left(M, \overrightarrow{\omega_{i}}\right) \vec{N} \cdot \overrightarrow{\omega_{i}} d \overrightarrow{\omega_{i}}$ where $\vec{\omega}_{r}$ is the direction of the reflected light and $\overrightarrow{\omega_{i}}$ the direction of the incident light.

In ${ }^{6}$, a color chart known as the Macbeth Table was proposed. Each color plate is given as a spectral reflectance curve (see Figure 2). This notion corresponds to the integration other an hemisphere of the flux re- flected by a material illuminated under normal incidence. Therefore, it is the integration of the BRDF of the material, with $\vec{\omega}_{i}=\vec{N}$.

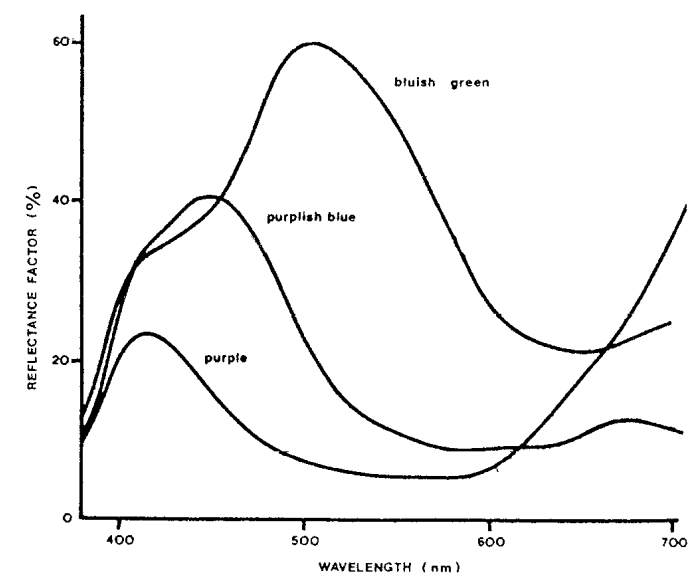

Figure 2: Some spectral reflectance curves from ${ }^{6}$

For a given point, and a lambertian type reflection, let us denote by $R(\lambda)$ the spectral reflectance of the surface and by $S_{i}(\lambda)$ the relative spectral power of light at normal incidence. Then the spectrum of the reflected light is defined by: $S_{r}(\lambda)=S_{i}(\lambda) R(\lambda)$. Such a reflection corresponds to a filtering operation.

Spectral rendering techniques may be arranged into three categories: Ray tracing, radiosity and Monte Carlo methods, which are more or less complementary. Since this topic is beyond the scope of our paper, we suggest the interested reader refers to ${ }^{4}, 7$ or ${ }^{8}$.

In conclusion, the important fact to emphasize is that, at the end of the rendering step, a spectrum has been computed by some algorithm for each pixel (i, j) of the screen.

\section{Computation of $X Y Z$ tristimulus values from a spectrum}

Light, as an electromagnetic wave, has to be described by its energetic spectrum. But studies in human vision showed that color may be represented in a three dimensional vector space. Many different color spaces exist, each of which being characterized by the choice of three color matching functions defined on the visible wavelength domain and independent from display devices.

Let us consider a spectral distribution of energy; its trichromatic components $P_{i}, 1 \leq i \leq 3$, are obtained by the formulas:

$$
P_{i}=\int_{\lambda_{\text {inf }}}^{\lambda_{\text {sup }}} S(\lambda) \sigma_{i}(\lambda) d \lambda
$$


where $\lambda_{\text {inf }}$ and $\lambda_{\text {sup }}$ are the bounds of the visible wavelength domain, and $\sigma_{i}(\lambda), 1 \leq i \leq 3$, are the color matching functions of the color space in which the calculation is made. The $X Y Z$ color space, introduced by the CIE (Commission Internationale de l'Eclairage) in 1931, is one of the most significant. This standardized space is defined by a set of three color matching functions $\bar{x}, \bar{y}$ and $\bar{z}$, represented in Figure 3. These functions are always positive and $\bar{y}$ corresponds to the photopic visibility function $V$ of the human eye. Since it is standardized, this color space turns out to be very useful for color conversion problems.

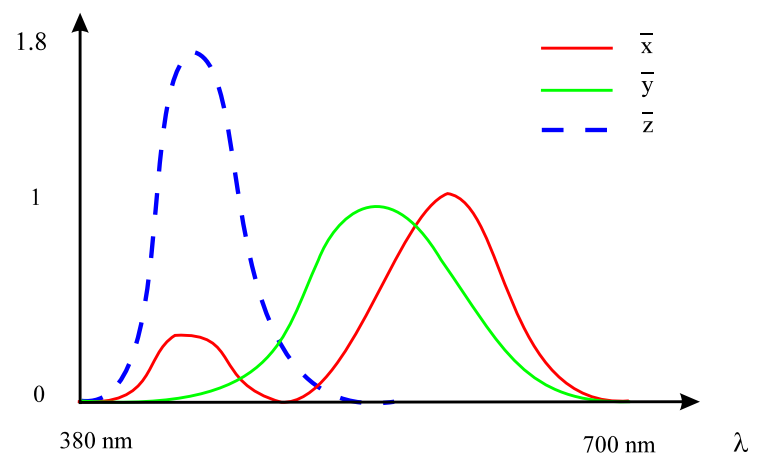

Figure 3: The color matching functions of the 1931 CIE XYZ color space

$X Y Z$ tristimulus values are obtained by the following calculation:

$$
\begin{aligned}
& X=K \int_{\lambda_{\text {inf }}}^{\lambda_{\text {sup }}} S_{r}(\lambda) \bar{x}(\lambda) d \lambda \\
& Y=K \int_{\lambda_{\text {inf }}}^{\lambda_{\text {sup }}} S_{r}(\lambda) \bar{y}(\lambda) d \lambda \\
& Z=K \int_{\lambda_{\text {inf }}}^{\lambda_{\text {sup }}} S_{r}(\lambda) \bar{z}(\lambda) d \lambda
\end{aligned}
$$

where $K$ is a normalization coefficient.

If the spectrum $S$ is expressed in absolute power (Watts), then $K$ is taken equal to $683 \mathrm{lum} / \mathrm{W}$, and $Y$ is a luminance expressed in $l u m / m^{2} s t r\left(\right.$ or $\left.c d / m^{2}\right)$. Otherwise, and when the scene contains only one light source, this source is assigned a luminance value of 1 , and the $X Y Z$ coordinates are given by formulas (1) with:

$$
K=\frac{1}{\int_{\lambda_{\text {inf }}}^{\lambda_{\text {sup }}} S(\lambda) \bar{y}(\lambda) d \lambda}
$$

where $S(\lambda)$ is the light source spectrum.

Remark: Most of the time, the light source is attributed a luminance of 100 and thus all $Y$ coordinates may be seen as percentages (luminance factors).
We are now going to describe several methods to compute tristimulus values. Then we shall present a few results we obtained by testing these methods. Let us notice that for the rest of the paper:

- a visible wavelength domain defined by $\lambda_{\text {inf }}=380$ $\mathrm{nm}$ and $\lambda_{\text {sup }}=700 \mathrm{~nm}$ will be used;

- tristimulus values will be normalized with coefficient $K$ obtained in Equation (2).

\subsection{Sampling methods}

\subsubsection{Direct method (weighted ordinate)}

This method, the most usual one, samples the visible spectrum every one, five or ten nanometers, and then uses a Riemann type integration. For the tests described in section 3.4, spectra were sampled every $5 \mathrm{~nm}$. In the case of a spectrum with emission peaks (such as a neon tube or a fluorescent light), the power of each peak is spread on the $5 \mathrm{~nm}$ widthband which surrounds it. Therefore, the formulas used are:

$$
\begin{aligned}
& X=K \sum_{i=1}^{i=64} S\left(\lambda_{i}\right) \bar{x}\left(\lambda_{i}\right) \\
& Y=K \sum_{i=1}^{i=64} S\left(\lambda_{i}\right) \bar{y}\left(\lambda_{i}\right) \\
& Z=K \sum_{i=1}^{i=64} S\left(\lambda_{i}\right) \bar{z}\left(\lambda_{i}\right)
\end{aligned}
$$

This method gives good results but is very time and memory consuming. It requires 64 multiplications for the spectrum-reflectance product step, and 192 multiplications plus 189 additions for the integration step.

In the following, we shall consider this method as supplying the reference tristimulus values for our tests.

\subsubsection{Gaussian quadrature method}

The mathematical principle of the method is as follows ${ }^{9}$ : Let $f$ be a function defined on interval $[\mathrm{a}, \mathrm{b}]$ and $w$ a weighting function positive on $[\mathrm{a}, \mathrm{b}]$. Then: $\int_{a}^{b} f(x) w(x) d x=\sum_{i=0}^{n} H_{i} f\left(x_{i}\right)+R(f)$

Coefficients $H_{i}$ are given by: $H_{i}=\int_{a}^{b} l_{i}(x) w(x) d x$ where $l_{i}(x)=\prod_{j=0 j \neq i}^{n} \frac{x-x_{j}}{x_{i}-x_{j}}$ and abscissas $x_{0}, \ldots, x_{n}$ are the zeros of the $(n+1)$-order polynomial of a family of polynomials orthogonal to $w(x)$ on $[\mathrm{a}, \mathrm{b}]$.

$R(f)$, the residual term, is defined by: $R(f)=\frac{(b-a)^{2 n+1}(n !)^{4}}{((2 n) !)^{3}(2 n+1)} f^{(2 n)}(\xi)$ whith $\xi \in[a, b]$.

In relation with the problem addressed, this mathematical method has been used in two different ways, according to the choice of the color space. 
3.1.2.1. Use of the $X Y Z$ color space. Color matching functions $\bar{x}, \bar{y}$ and $\bar{z}$ are viewed as weighting functions. Weights $w_{x}, w_{y}$ and $w_{z}$ correspond to coefficients $H_{i}$ and wavelengths $\lambda_{x}, \lambda_{y}$ and $\lambda_{z}$ to abscissas $x_{i}$ and they just have to be pre-computed.

With this model, a spectrum-reflectance product requires $m x+m y+m z$ multiplications and an integration $m x+m y+m z$ multiplications plus $(m x-1)+$ $(m y-1)+(m z-1)$ additions.

3.1.2.2. Use of the $A C_{1} C_{2}$ space. The $A C_{1} C_{2}$ space was introduced by G. MEYER 10 and may be derived from the $X Y Z$ color space by a linear transformation. The axes of the $A C_{1} C_{2}$ color space have been chosen in such a way that they pass through the densest regions of tristimulus values, and that their intercorrelation is minimized. Three matching functions are obtained: $\bar{a}(\lambda)$ carrying the most important part of the information and corresponding to the photopic visibility function, $\overline{c_{1}}(\lambda)$ fitting with the $\mathrm{red} /$ green opponency, and $\overline{c_{2}}(\lambda)$ with the yellow/blue one. $m a>m c 1>m c 2$ must be respected to allow the best results.

Portions of matching functions $\overline{c_{1}}(\lambda)$ and $\overline{c_{2}}(\lambda)$ are negative on the visible wavelength domain, hence some orders of integration with the gaussian quadrature method may not be possible.

With this space, the cost of a spectrum-reflectance product is $m a+m c 1+m c 2$ multiplications. An integration requires $m a+m c 1+m c 2+9$ multiplications plus $(m a-1)+(m c 1-1)+(m c 2-1)+6$ additions to be performed (this cost includes the change to the $X Y Z$ color space with a $3 \times 3$ matrix product).

\subsection{Segmentation of the visible wavelength domain}

The main objective of this method ${ }^{11}$ is to take all the types of spectra into account, be they continuous or not. Similarly to the Gaussian quadrature, this method may be used with the $X Y Z$ color space, or with the $A C_{1} C_{2}$ one.

This method works with the following principle: The visible field of each light source in the scene is partitioned into a set of intervals, in two steps:

- the non-zero portions of the spectrum are extracted;

- for these intervals, peaks are isolated from the continuum through a gradient calculation.

To obtain the final set of bandwiths, all the sets associated to light sources are concatened with an operator OP defined as: If $\mathrm{a}$ and $\mathrm{b}$ are two sets of intervals then $\mathrm{OP}(\mathrm{a}, \mathrm{b})=$ concatenation $((a \cup b)-(a \cap b),(a \cap b))$.
From this final set of intervals, a series of wavelengths may be precomputed:

- if the current interval corresponds to a continuum, then a gaussian quadrature method is employed;

- else (it corresponds to an emission peak), a trapezoidal method is used.

Finally, a spectrum is represented by $m a+m c 1+$ $m c 2$ (or $m x+m y+m z$ ) wavelengths plus (if needed) mpeak other wavelengths. The spectrum-reflectance product is made, as usual, wavelength to wavelength.

With this method, the cost of a spectrumreflectance product is $m a+m c 1+m c 2+$ mpeak multiplications. An integration requires $m a+m c 1+m c 2+$ 3 mpeak +9 multiplications plus $(m a-1)+(m c 1-$ $1)+(m c 2-1)+3($ mpeak -1$)+6$ additions.

\subsection{Projection on a set of basis functions}

\subsubsection{Use of polynomial functions}

Raso and Fournier 12 propose to use piecewise cubic polynomials to represent spectral power distributions. All spectral data are approximated by two spans of a cubic polynomial with the spectrum divided at $550 \mathrm{~nm}$. They use a least squares algorithm to determine the coefficients for the polynomials. Interreflection computation are thus reduced to polynomial multiplications. To avoid obtaining very high degrees for the final polynomial, the degree of the polynomials is kept constant by using a degree reduction technique based on Chebyshev polynomials. To finally obtain $X Y Z$ tristimulus values, the color matching functions $\bar{x}(\lambda), \bar{y}(\lambda)$ and $\bar{z}(\lambda)$ are also represented by a cubic piecewise polynomial. With this solution, the costs are constant: 80 multiplications and 58 additions for a spectrum-refletance product, 46 multiplications and 30 additions for an integration.

\subsubsection{Functions obtained by a characteristic vector analysis}

The method presented in ${ }^{13}$ has for main advantage to take the scene to be rendered into account, which should allow to adapt correctly to the future data to be processed.

The principle on wich the method works is the following: From a family of spectral power distributions known to be representative of the scene, a characteristic vector analysis is performed providing $m$ vectors: $E_{1}(\lambda), \ldots, E_{m}(\lambda)$. These vectors $E_{i}(\lambda)$ are the eigenvectors associated to the $m$ greatest eigenvalues of the covariance matrix of the inital vectors.

The choice of a representative family can be solved in the following way: if the scene is made up of a light 
source $S$ and $n$ materials with reflectance $R_{1}, \ldots, R_{n}$, then spectra $S, S * R_{i}, 1 \leq i \leq n, S * R_{i} * R_{j}, 1 \leq$ $i, j \leq n$. . may be taken into account.

After this pre-processing step, each spectral distribution is represented by its $m$ coordinates in the basis: $\left(E_{1}(\lambda), \ldots, E_{m}(\lambda)\right)$, and reflectance $R(\lambda)$ takes the form of an $m \times m$ matrix $\left(R_{i j}\right)$, with: $R_{i j}=\int_{\lambda_{\text {inf }}}^{\lambda_{\text {sup }}} R(\lambda) E_{i}(\lambda) E_{j}(\lambda) d \lambda$

Finally, for the integration step, a three lines and $m$ columns matrix $T$ has to be precomputed, with:

$$
\begin{aligned}
T(1, i) & =\int_{\lambda_{\text {inf }}}^{\lambda_{\text {sup }}} E_{i}(\lambda) \bar{x}(\lambda) d \lambda \\
T(2, i) & =\int_{\lambda_{\text {inf }}}^{\lambda_{\text {sup }}} E_{i}(\lambda) \bar{y}(\lambda) d \lambda \\
T(3, i) & =\int_{\lambda_{\text {inf }}}^{\lambda_{\text {sup }}} E_{i}(\lambda) \bar{z}(\lambda) d \lambda
\end{aligned}
$$

In conclusion, this method, which is entirely linear (this idea was already presented in ${ }^{14}$ ) requires $m^{2}$ multiplications plus $m(m-1)$ additions for a spectrum-reflectance product, and $3 m$ multiplications plus $3(m-1)$ additions for an integration.

\subsection{Tests}

Remind that the goal of the methods presented in sections $3.1,3.2$ and 3.3 is to compute tristimulus values providing non perceptual errors and requiring as little operations as possible. To evaluate these methods, we used:

- Three types of light sources: The standard illuminants C and D6500 with continuous spectra, and a fluorescent source with four peaks;

- Twenty-four reflectances taken from the Macbeth Color Checker chart ${ }^{6}$, which have been developed to facilitate the quantitative or visual evaluation of color reproduction processes used in photography, television, and printing;

- The Luv color space, supposed to be uniform, to perform error calculation. In this space, an error lower than 1 may be considered as non-perceptible for a human eye.

We used the following procedure: For a couple of a given light source and a given reflectance, a set of tristimulus values is computed with the reference method. Then for a given algorithm and an order $m$, the same set of tristimulus values is estimated and the distance between the two tristimulus values is computed in the Luv color space.

We computed the average Luv error (on the twentyfour reflectances of the Macbeth Color Checker) as a function of the cost per operation to accomplish a spectrum-reflectance product or an integration. The diagrams showing the complete results of these tests may be consulted in ${ }^{15}$. Let us only consider two of them to illustrate our remarks.

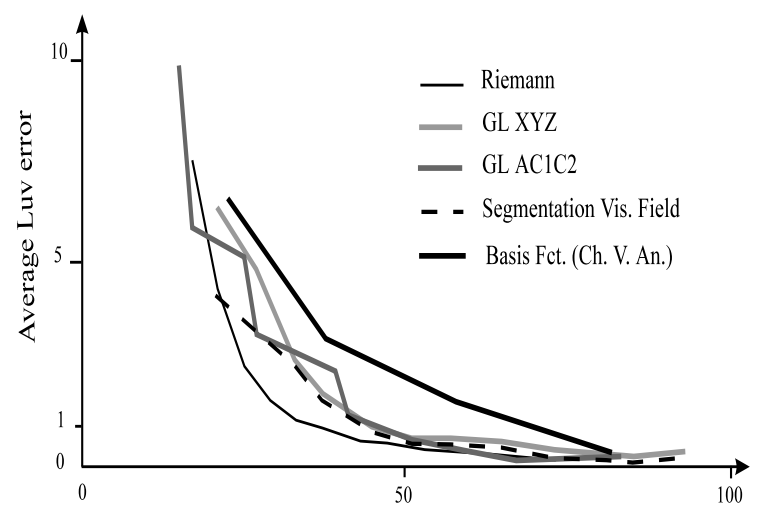

Figure 4: Cost of a spectrum-reflectance product with light source: $C$

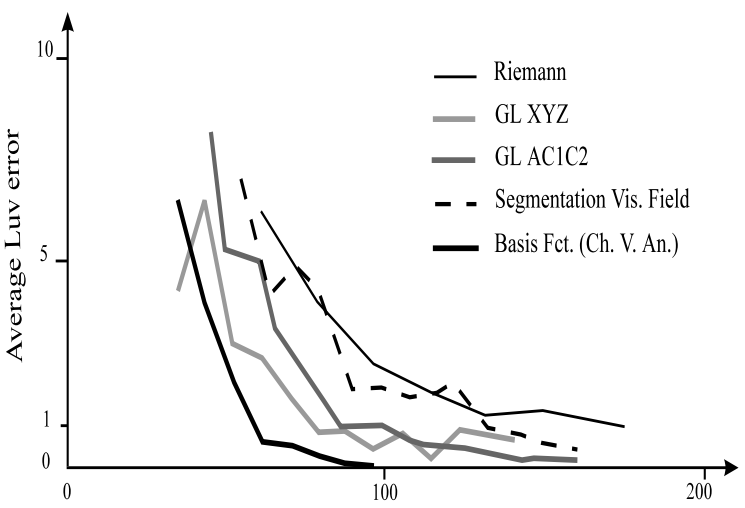

Figure 5: Cost of an integration with light source: D6500

- For CIE Standard Illuminants C and D6500, the Riemann based algorithm (using less evenly spaced points than the reference method) provides the best results for spectrum-reflectance products, whereas the projection on a set of basis functions obtained by a characteristic vector analysis is the cheapest for integration;

- The use of the $A C_{1} C_{2}$ color space does not seem to bring a lot of efficiency. As we need $X Y Z$ coordinates at the end of the process, we must use a $3 \times 3$ matrix product to come back to the desired color space;

- For the fluorescent source, the segmentation method and the projection on a set of basis functions obtained by a characteristic vector analysis are the 
only two methods capable of handling this case. For the spectrum-reflectance products, the first solution seems to be preferable. For the integration, the second one is leading;

- Gauss-Legendre methods are at disadvantaged when compared with the other methods by the fact that every color matching function requires a family of wavelengths and a family of weights. Borges 16 suggested a solution to solve this problem;

- By using the twenty-four reflectances from the Macbeth Color Checker to build the initial family ( $S$, $\left.S * R_{i}, 1 \leq i \leq 24\right)$, the characteristic vector analysis provides a basis composed of 6 vectors for an average Luv error lower than 1;

- For the solution with projection on polynomial functions, we found that degree four polynomials are required to produce an Luv error lower than 1 after light has been reflected once from surfaces in the scene.

\section{The Color Reproduction Function}

\subsection{Introduction}

Let us remind (cf. section 1) that the theoretical purpose of the Color Reproduction Function (CRF) is to supply data to a frame buffer in such a way that when these data are displayed, they produce the same sensations for the monitor observer as if he were facing the real scene. Adopting a black box representation for this CRF, we have:

- as input, computed $X Y Z$ values which are assigned to each pixel;

- as output, numerical $R G B$ values (bounded by 0 and 255).

Figure 6 shows that the content of the CRF black box is the following:

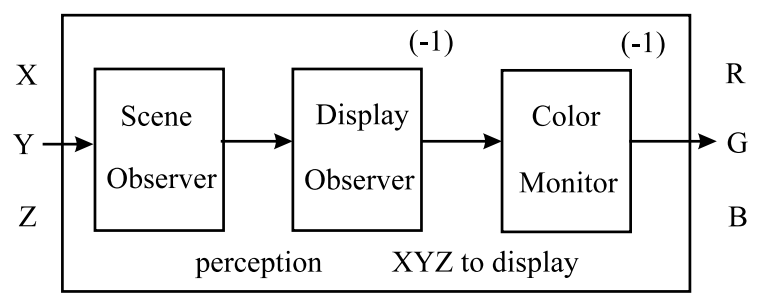

Figure 6: Diagram of a Color Reproduction Function

In the next section, we shall first specify what we mean by an observer model. Then we shall survey the different solutions allowing to calculate the tristimulus values to be displayed that have already been suggested in the literature. Finally, some ideas about future extensions will be given.

\subsection{An observer model}

First, the difficulty to model human color vision has to be emphasized. It seems that the achievement of a global model, concerning any type of situation, is still out of reach. Different vision mecanisms have been isolated and studied during specific experiments. Unfortunately, these different phenomena all take place at the same time for complex environments, interfering and hiding each other. Therefore, no formalization may claim to universality.

Despite these remarks, we shall try to define the most general observer model, while knowing that a part of empirism must be introduced. This means that the idea of a completely automatic CRF must be forgotten. The real display observer should interactively adjust some parameters, working by trial-and-error on the complete image or more simply on a test card.

\subsubsection{Introduction}

First, we have to keep in mind that the $X Y Z$ color system is only valid for diurnal situations, which correspond to photopic vision, with luminance bounds varying approximatively from some $c d / \mathrm{m}^{2}$ to $10^{8} \mathrm{~cd} / \mathrm{m}^{2}$. In nocturnal vision (or scotopic, with luminances ranging from $10^{-6}$ to $10^{-3} \mathrm{~cd} / \mathrm{m}^{2}$ ), no color notion exists. Therefore, just one $Y$ coordinate needs to be calculated, with the formula:

$$
Y=K_{m}^{\prime} \int_{\lambda_{\text {inf }}}^{\lambda_{\text {sup }}} S(\lambda) V^{\prime}(\lambda) d \lambda
$$

where $K_{m}^{\prime}=1700 \mathrm{~cd} / \mathrm{W}$ and $V^{\prime}$ is the luminous efficiency function for scotopic vision. Its maximum is shifted toward blue in relation to the photopic function $V(507 \mathrm{~nm}$ vs $555 \mathrm{~nm})$.

Furthermore, an intermediary vision (mesopic) exists for luminances in the range of $10^{-3}$ to some $\mathrm{cd} / \mathrm{m}^{2}$, and is characterized by two main phenomena. First, by increasing the average luminance level, there is a progressive shifting of the visibility function from $V^{\prime}$ to $V$, which is called the Purkinje shift. Secondly, under the same conditions, the sensibility threshold of the different cones are gradually reached, and color notion arises then.

Let us notice that in ${ }^{17}$, an attempt was made to simulate visual adaptation over a very wide luminance scale (covering all three types of vision, see section 4.2.4).

In conclusion, the average luminance level of a scene must carefully be taken into account to determine in which domain to operate. For the rest of this paper, only diurnal vision will be considered.

The second point to be evoked in this introduction is the fact that the analysis level is not the same everywhere in the vision field. It is well-known that visual 
acuity (related to the distribution of photoreceptors), is maximized around the fovea. Hence, when an observer looks at a particular object, this means that he focuses his attention on this object, directing his fovea to better discriminate details. In ${ }^{18}$, Burt reminds us that acuity falls roughly in inverse proportion to the visual angle from fovea. However, vision field is most of the time divided in several areas, instead of considering a continuous decrease. In ${ }^{19}$, five areas are proposed: Adapting, surround, background, and local induction field, plus the color element taken into account. In this survey, we shall only take two areas into account: The focus area (extending two degrees around the aim), and the peripheral area (or background, contributing to the global adaptation).

Finally, we shall try in the following to separate as much as possible the luminance analysis from the chromatic one.

\subsubsection{Luminance analysis}

In this case, the local analysis area is the zone where simultaneous constrasts take place. To better discriminate between objects, the eye naturally emphasizes differences. This can be explained physiologically by the fact that an excited receptor tends to inhibit its neighbors. A well-known example of simultaneous contrast is given in figure 7 .

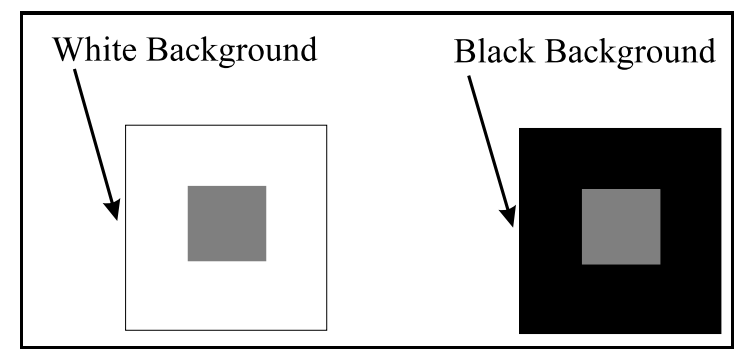

Figure 7: Squares at the center have the same luminance; yet the left one seems darker than the right one

Moreover, there is a general adaptation of the eye. This phenomenon streches over a rather consequent period of time. It takes several minutes to go from darkness to light, and from fifteen to twenty minutes for the opposite way. A physiological explanation for this adaptation is given in ${ }^{17}$. It relies on four facts:

- the change in diameter of the pupil;

- the existence of two types of photoreceptors, rods and cones, each of them with a preponderant activity either in scotopic or photopic vision;

- the bleaching and the regeneration of photopigments;

- neural processes, the gain of each neuron being adapted by retroaction to the transmitted signal.
Once luminance adaptation is performed, we now need to deal with the notion of brightness. In ${ }^{20}$, it is defined as follows: "Brigthness is the attribute of a visual sensation according to which...the area in which the visual stimulus is presented appears to emit more or less light".

Different laws based on experiments attempt to link this subjective sensation to luminance. It may be chronologically quoted:

- Weber-Fechner's law (see ${ }^{21}$ ).

According to Weber, the ratio of perceived difference luminance on luminance is constant, so: $\frac{\Delta L}{L}=$ cst. Weber-Fechner's law introduces the notion of sensation proportional to Weber's ratio, that is to say: $\Delta S=k_{1} \frac{\Delta L}{L}$. The integration of the last formula yields: $S=k_{2} \ln L+k_{3}$.

This logarithmic variation linking luminance to sensation is a first good formalization of the phenomenon. Nevertheless, its validity field seems to be restricted to luminances ranging from some $\mathrm{cd} / \mathrm{m}^{2}$ to about $500 \mathrm{~cd} / \mathrm{m}^{2}$;

- Stevens and Stevens's law ${ }^{22}$.

Let us consider the following experiment: An observer is asked to suit his eye to a white background with luminance $L_{B}$. Then, he is briefly put in presence of a gray target with luminance $L$, against the previous background. Brightness is then assessed. Formally: $B=10^{\beta} L^{\alpha}$ with $\alpha=0.4 \log L_{B}+2.92$ and $\beta=-0.4\left(\log L_{B}\right)^{2}-2.584 \log L_{B}+2,0208(\mathrm{~B}$ is here expressed in Brils);

- Bodmann, Haubner and Marsden's law (see ${ }^{23}$ ). First, instead of considering luminance as a stimulus for brightness, the authors recommend to use equivalent luminance. As the proposed law is achromatic, the notion of equivalent luminance allows to take colored lights into account, correcting the effect that chromatic coordinates could introduce. Thus, for a given luminance and a given hue, the more a color is saturated, the more luminous it appears. Therefore, Bodmann, Haubner and Marsden suggest to use the following formula: $L_{e q}=L 10^{C(x, y)}$ where $C(x, y)=0.256-0.184 y-2.527 x y+4.656 x^{3} y+$ $4.657 y^{4}$. Finally, for a target with a width of 2 degrees and with equivalent luminance $L$, and for a background luminance $L_{B}$, brightness is defined as follows: $B=C(\phi) L^{n}-B_{0}\left(L_{B}, \phi\right)$ with $B_{0}\left(L_{B}, \phi\right)=$ $C(\phi)\left(S_{0}(\phi)+S_{1}(\phi) L_{B}^{n}\right)$ where $n=0.31$. For $\phi=2$ degrees: $C(\phi)=22.969, S_{1}(\phi)=0.07186$ and $S_{0}(\phi)=0.24481$.

Let us notice that these relations are issued from experiments where situations are rather simple. For a complex image, the exact notion of background luminance must be well-defined. 


\subsubsection{Chromatic analysis}

At a local (and immediate) level, there is once again a simultaneous contrast phenomenon between opponent colors (red/green and yellow/blue). For example, a gray target put against a red background will tend to seem greenish.

At a more global level, there is another mechanism called chromatic adaptation which is not instantaneous but nevertheless quick. To illustrate this phenomenon, we can give the example of a monochromatic television screen, which seems bluish when seen by night, from outdside and through a window. In this situation, prevailing illuminants (for example, streets lamps) determine the observer's adaptation and the criterion for white. As the television set is only a small and distant object, it seems blue. On the other hand, when this screen is watched at a usual distance, it dominates most of the vision field and becomes the criterion for white. Therefore, it appears in gray scale.

The first formalization of this phenomenon was given by Von Kries in 1878 (cf. ${ }^{24}$ ), and still provides a good approximation. It postulates that visual responses are proportional to the physical stimulation of each of the three sets of receptors, and that only the ratios of the coefficients of proportionality change with adaptation.

More formally, let $X^{\prime} Y^{\prime} Z^{\prime}$ be the tristimulus values of a given color for a given illuminant and let $X Y Z$ be the tristimulus values of a color which seems identical to the first one for a second illuminant. If we assume we now have a set of primaries, the tristimulus values of the two previous colors will be denoted $R G B$ and $R^{\prime} G^{\prime} B^{\prime}$. Then, we can find three coefficients $K_{R}, K_{G}$ and $K_{B}$, only depending on illuminants, such that:

$$
\begin{aligned}
R^{\prime} & =K_{R} R \\
G^{\prime} & =K_{G} G \\
B^{\prime} & =K_{B} B
\end{aligned}
$$

The values of these coefficients come from experiments. They are computed in such a way that they minimize the least squares error in the $X Y Z$ color space for a family of tristimulus couples paired for a given change of illuminant. Chromaticities of the most used red and green primaries are Judd's $P$ and $T$ points: $\left(x_{r}=0,747 ; y_{r}=0,253\right),\left(x_{b}=0,1785\right.$; $\left.y_{b}=0\right)$. According to Mac Adam's work, the most suitable green primary would have coordinates: $\left(x_{g}=\right.$ 0,$\left.75 ; y_{g}=-1,75\right)$. Let us notice that these primaries are partly outside of the visible domain.

These laws allow to predict whether there will be color constancy for an illuminant change or not.

\subsubsection{Known solutions}

To our knowledge, papers on this subject are only concerned with gray scale images. In this case, Reproduction Function Color (RFC) is reduced to a Tone Reproduction Function (TRF).

Historically, the first paper to describe the whole process (with an observer and a monitor model) was presented by Tumblin and Ruschmeier ${ }^{25}$. Luminance is linked to brightness following Stevens and Stevens's law.

For the scene observer: $B_{S}=10^{\beta_{S}} L_{S}^{\alpha_{S}}$, where $\beta_{S}$ and $\alpha_{S}$ depend on the scene background luminance $L_{S B} . L_{S B}$ is deduced from perceived luminances of the scene by formula: $\log L_{S B}=E\left(\log L_{S}\right)+0.84$, where $E$ is the expected value.

For the display observer: $B_{D}=10^{\beta_{D}} L_{D}^{\alpha_{D}}$. The determination of the background luminance for the displayed image raises a paradox, even in the simplest case where the observer is located in a dark room. Actually, background luminance cannot be obtained as an average of displayed luminances because their computation depends on it. Therefore, its value must be arbitrarily fixed. Generally, it is defined as:

$$
L_{D B}=\left(L_{\text {maxdisplay }}\right) / C s t .
$$

For Tumblin and Rushmeier, this constant is assigned the value $\sqrt{C_{\max }}$, where $C_{\max }$ (typically equal to 35 ) is the ratio of the maximum luminance displayable by a monitor to the minimum one. Therefore, we have $L_{D B}=\sqrt{L_{\text {maxdisplay }} L_{\text {mindisplay }}}$

If brightness is supposed to be the same for both observers, then the following final relation between scene and display luminances is obtained:

$$
L_{D}=L_{S}^{\frac{\alpha_{S}}{\alpha_{D}}} 10^{\frac{\beta_{S}-\beta_{D}}{\alpha_{D}}}
$$

In ${ }^{26}$, D'Amico and Taugourdeau follow the same process but use two different brightness laws (for comparison purposes). The first law is still Stevens and Stevens's. The second law is: $B=23 \log L-$ $5.5 \log \left(L_{B}\right)-1.6$.

The assumption of brightness equality leads to the following relation:

$$
L_{D}=L_{S}\left(\frac{L_{D B}}{L_{S B}}\right)^{\left(\frac{5.5}{23}\right)}
$$

For comparative tests, background luminances are fixed as follows:

$L_{S B}=E\left(L_{S}\right)$

$L_{D B}=L_{\text {maxdisplay }} / 10$.

A conclusion from the test of these two TRF is that the one using Stevens and Stevens's law appears to perceptually behave better. However, none of them seems capable of correctly dealing with scenes with a 
high dynamics. Depending on the value calculated for background scene luminance, some parts of the picture are over (or under) exposed.

An area approach for a non-uniform TRF was also tested. A threshold value is determined after investigation of the luminance histogram. It allows to separate bright from dark areas in the image, and to compute two different background luminances (LSBbright, LSB-dark). According to whether the current pixel belongs to one area or to the other, it is applied the corresponding TRF.

This idea has also been suggested in ${ }^{27}$ and ${ }^{28}$. Stating that a uniform TRF cannot process all the cases, the authors propose to evaluate for each current pixel an adaptation level by studying its neighborhood. Let us notice that these methods do not use any observer model. In fact, their main goal is to reduce luminance dynamics to monitor possibilities, keeping as much as possible an idea of the initial image perception.

If these methods are of lower claim, they seem efficient, and at least easy to implement. In particular, very few parameters have to be adjusted.

In ${ }^{29}$, Ward tries to find out a linear relation between scene and display luminances, so that: $L_{D}=$ $m L_{S}$. Rather than using the brightness equality hypothesis, a match is made between minimum noticeable differences. These ones correspond to allowed luminance difference $L$ of a target against a background with luminance $L_{B}$, so that no difference may be perceived.

At the beginning of the 70's, Blackwell gave the following relation: $\Delta L\left(L_{B}\right)=0.054\left(1.219+L_{B}^{0.4}\right)^{2.5}$

Ward is hoping to set: $\Delta L_{D}=m \Delta L_{S}$.

So: $m=\left(\frac{1.219+L_{D B}^{0.4}}{1.219+L_{S B} 0.4}\right)^{2.5}$

and $L_{D}=\left(\frac{1.219+L_{D B}^{0.4}}{1.219+L_{S B} 0.4}\right)^{2.5} L_{S}$

In order to fix the background scene luminance value, Ward suggests an uncommon method. He assumes the scene observer focuses his attention on a certain point. The area surrounding this point contributes to set his adaptation level still more. Therefore, $L_{S B}$ is obtained as the luminance average on that area. This permits to distinguish a luminance level well, even if darker areas tend to be under-exposed, and brighter ones over-exposed.

On the other hand, display background luminance is more usually found as: $L_{D B}=L_{\text {maxdisplay }} / 2$.

In ${ }^{17}$, as mentioned earlier, visual adaptation is simulated over a wide range of luminances. For this purpose, Ward's algorithm is used. This first relation, valid in photopic conditions, is then extended to scotopic ones. Finally, by interpolating between these two formulations, the mesopic field may also be taken into account.

\subsubsection{Proposal for a methodology}

For the luminance processing, we presented already known solutions. A very useful extension of these methods would be to allow them to take color images into account. For this, we believe the notion of equivalent luminance should be used in order to correct the influence of chromatic coordinates. Bodmann argued that his brightness law generalizes the results which can be obtained with other laws. Thus, the formula described in section 4.2.2 should be used. Furthermore, on the local analysis area, simultaneous contrast should be taken into account to be corrected.

The performances of the proposed TRF mostly rely on the way background luminances are calculated. These ways often seem arbitrary. An already suggested solution to this problem would be the use of test cards, or the possibility to interactively modify some CRF parameters until satisfaction of the observer of the image.

Otherwise, we believe that to really deal with color images, the notion of chromatic adaptation should be introduced in CRF. For both observers, the prevailing type of illuminant simply needs to be determined. For the scene observer, this could be rather complex if different types of light sources (a natural source and an artificial one, or an incandescent lamp and a fluorescent tube) coexist. On the other hand, if the display observer is located in a dark room, his criterion for white is given by the screen, without doubt.

We may observe here that the two observers are set in very different situations. The field of vision of the scene observer is entirely occupied by the scene, but it is far from being the case for the screen observer. Thus, the screen size and the distance of vision must be assessed for the screen observer. In the same way, several questions should be answered about the immediate surrounding: Is the room dark? Else, what kind of illuminant is used? How high is the reflectance screen? What is the background wall color? What is the frame screen color?

To sum up, an improved model of vision should take both types of adaptation presented before into account. Unfortunately, the straightforward idea of using one law one after another for each adaptation in the $X Y Z$ color space is certainly not a good one. First, reversing the order of use of these formulas would not lead to the same results. Secondly, the $X Y Z$ color space is not adequate to perform this type of calculation since its axes are highly intercorrelated. In fact, formalizing the combination of both phenomena does not seem to be an easy task. 
However, let us repeat one last time that no vision model may claim to universality. In particular, the fact that the real display observer has to evaluate the fidelity level in comparison with a scene he has never seen, has to be emphasized. The subjective side of this experiment is strengthened by the fact that the memory of the observer, his imagination or even his own cultural knowledge have to be taken into account.

\subsection{A monitor model}

At this stage of the Color Reproduction Function, $X Y Z$ values have been computed. These values must be mapped to $R G B$ values which will be displayed on a given monitor. We are now aiming to develop this last step by describing the monitor calibration process and by taking the limitations of our current monitors into account.

\subsubsection{Color monitor calibration}

The relation linking numerical $R G B$ values to emitted $X Y Z$ tristimulus has to be determined.

For a low requirement level, data provided by manufacturers may be consulted. But, all cathode ray tubes are not rigorously identical and variations may arise with time. So, for advanced experimentations, the monitor used should be carefully calibrated.

Measurement quality of this operation depends of course mainly on the equipment used. Cowan ${ }^{30}$ suggested a fast process only requiring a lightmeter. First, he suggests to set up the monitor exactly as it will be when used. This means taking its spatial position, its contrast, its brightness, the relative gains of its three guns... into account. Then, he contents himself with the chromaticities and the white point furnished by the manufacturer.

The relation linking tristimulus to phosphor excitations is a simple matrix product:

$$
\left(\begin{array}{c}
X \\
Y \\
Z
\end{array}\right)=\left(\begin{array}{ccc}
X_{r} & X_{g} & X_{b} \\
Y_{r} & Y_{g} & Y_{b} \\
Z_{r} & Z_{g} & Z_{b}
\end{array}\right)\left(\begin{array}{c}
E_{r} \\
E_{g} \\
E_{b}
\end{array}\right)
$$

Finally, knowing that gun voltages are related to excitations by a non linear formula, its parameters may be assessed (adjusting them to measure curves), so that non-linearity may be corrected. This is the so called gamma-correction.

Different laws may be used for measure adjustement:

- The most usual one: $E_{a}\left(V_{a}\right)=\left(V a / V_{a \max }\right)^{\gamma_{a}}$ (where $a=r, g$ or $b$ );

- Cowan proposed in 30 : $\ln E_{a}\left(V_{a}\right)=A_{a} \ln \left(V_{a} / V_{a \max }\right)+B_{a} \ln ^{2}\left(V_{a} / V_{a \max }\right)$;
- It may be suggested to use: $\ln E_{a}\left(V_{a}\right)=$ $A_{a} \ln \left(V_{a} / V_{a \max }\right)+B_{a} \ln ^{2}\left(V_{a} / V_{a \max }\right)+C_{a}$.

Thus, $R G B$ values may be deduced from $X Y Z$ coordinates by gamma correction and inversion of the matrix in (3).

Brainard 31 uses a spectrophotometer to measure emission spectra of phosphors with enough accuracy to take emission rays into account. With this means, a more rigorous process may be followed. First, assumptions on the performance of the monitor are made. Then, adequate parameters are evaluated. Finally, validation of the assumptions is assessed.

The most frequently made assumptions are:

1. Phosphor constancy: Relative spectral power emitted by a phosphor does not depend on excitation;

2. Phosphor independence: Phosphor excitation only depends on its input values, not on the values of its neighbors;

3. Spatial independence: The amount of light emitted at a location only depends on the input values at this location. This is a notion of independence between pixels;

4. Spatial inhomogeneity through the surface of the screen, which implies the use of a correcting term $K\left(x_{p i x}, y_{p i x}\right)$ for absolute emitted power.

If these assumptions are valid, then phosphor chromaticities, the white point of the screen, the parameters of gamma-correction and those of spatial inhomogeneity just have to be measured. This last phenomenon, very often forgotten, does not seem to be negligible. Brainard measured differences between the center and the sides of the screen up to $20 \%$ of absolute power. This is explained by the fact that electron beams reach peripheral pixels oblically. Finally, Brainard raised the question of temporal stability. For this purpose, he recalibrated the same monitor two months after the initial experiment. Fortunately, no important change was noticed.

To avoid to spoil the work done before, this monitor calibration step requires a great care. At the end of this step, $R G B$ values that should theoretically be displayed are known. But, nothing ensures that they belong to the field of the displayable colors of the monitor.

\subsubsection{Monitor limitations}

There are two different types of limitations. First, even when connected to a 16 million colors frame buffer, a monitor does not cover all human colorimetric capacities. In fact, phosphor chromaticities are not enough saturated to provide such possibilities. The set of all chromaticities displayable by a color monitor is contained in a $R G B$ triangle. This set is called the gamut 
of the monitor. As can be seen in Figure 8, it is included in the human locus.

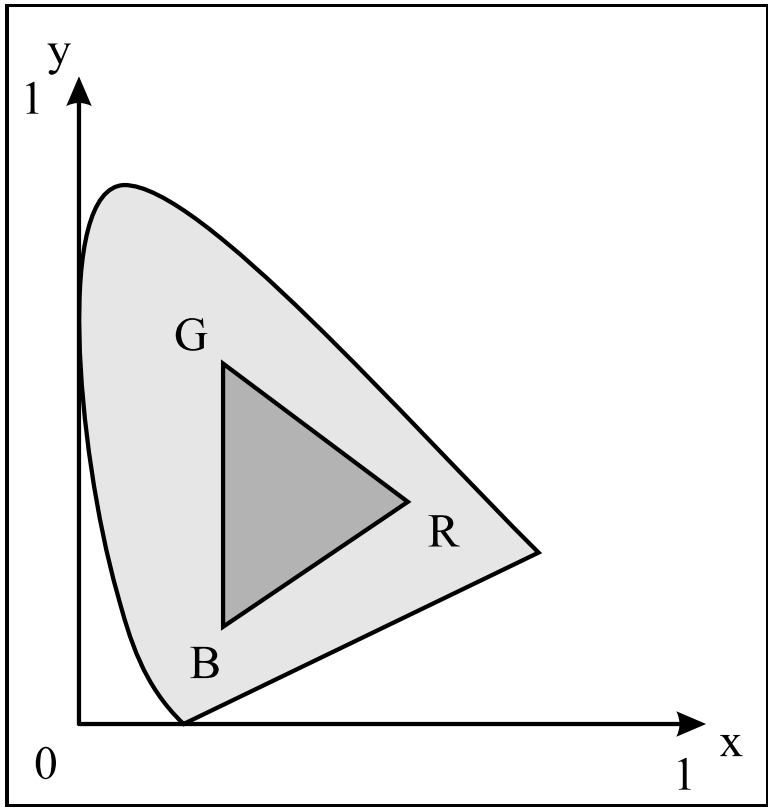

Figure 8: Chromaticities displayable by a color monitor

The second limitation refers to luminances. For most CRTs, maximum luminances are in the region of $100 \mathrm{~cd} / \mathrm{m}^{2}$.

Taking up a three-dimensional vision of the problem, it can even be emphasized that these two types of limitations are linked. Actually, monitor's possibilities are orchestrated around the gray axis, (which contains, in the $R G B$ color space, all points with coordinates $[N, N, N], 0 \leq N \leq 255)$. This implies that the most saturated colors (the farthest from this axis) are not the most luminous. On the other hand, highest (or lowest) luminances correspond to quasiinsaturated colors (see Figure 9).

$R G B$ values obtained in section 4.3 .1 may be out of the displayable colors of the monitor. The two cases described above may occur. First, the computed vectors may have a direction which does not intersect the $R G B$ cube (problem on chromaticities). Secondly, their magnitude may be too important (problem on luminance). Of course, both cases may take place at the same time.

The problem is to take back all these coordinates inside the $R G B$ cube. For this, a mapping operation is required. Let us notice that this can be done either in the $R G B$ computer color space, or in the $X Y Z$ space, because the order between the change from $X Y Z$ tris-

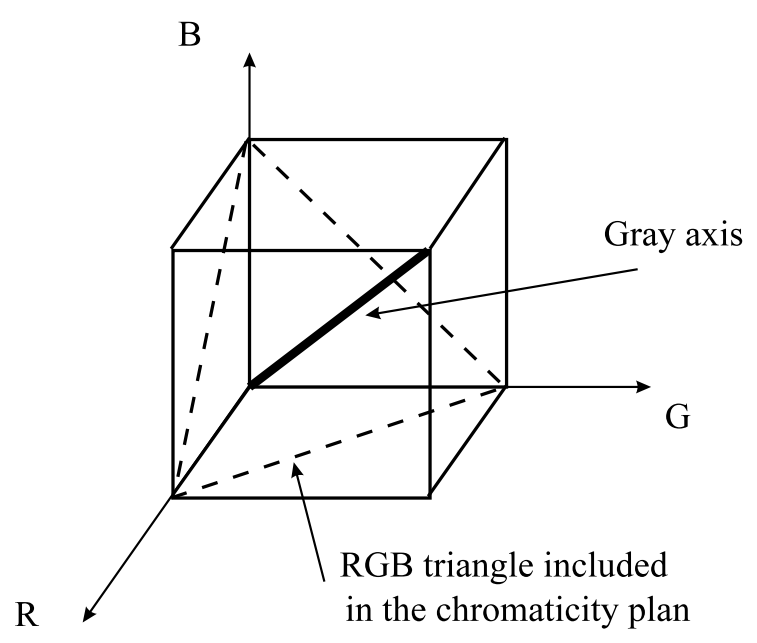

Figure 9: Links between colors and luminances

timulus to $R G B$ coordinates and the mapping operation may be reversed.

There are two ways to perform mapping on a monitor gamut. The first one is clipping, which means keeping coordinates inside gamut unchanged while projecting the others on its boundary. The second way is the use of a global transformation such as a translation, a rotation or a scaling, on the set of coordinates.

- Working in the RGB space

Clipping can be done very easily. For example, values greater than 255 are changed to 255 , and negative ones to zero.

In ${ }^{32}$, Smith manages to define a global transformation. He starts from an experimental fact which may be expressed in the following way: "Objects of high luminance appear whiter, compared to their environment, than chromaticity would suggest". The proposed transformation requires two steps. First, let us consider the unit $R G B$ cube. Let $\theta_{0}$ be the angle between the gray axis and a given set of values $R_{0}, G_{0}, B_{0}$. This set is then shifted towards the gray axis (refer to Figure 10) so that its new angle $\theta_{1}$ is:

$$
\theta_{1}=\theta_{0}\left(1-\left(\frac{Y}{\gamma Y_{\max }}\right)^{\beta}\right)
$$

$Y_{\max }$ is the luminance of the most luminous object in the scene, except light sources. As this object is not necessarily white, a parameter $\gamma$ must be introduced.

The second step lies in scaling new values $R_{1}, G_{1}$, $B_{1}$ with the following laws:

$$
\begin{aligned}
R_{2} & =R_{1}\left(Y / Y_{\max }\right)^{\alpha} \\
G_{2} & =G_{1}\left(Y / Y_{\max }\right)^{\alpha}
\end{aligned}
$$




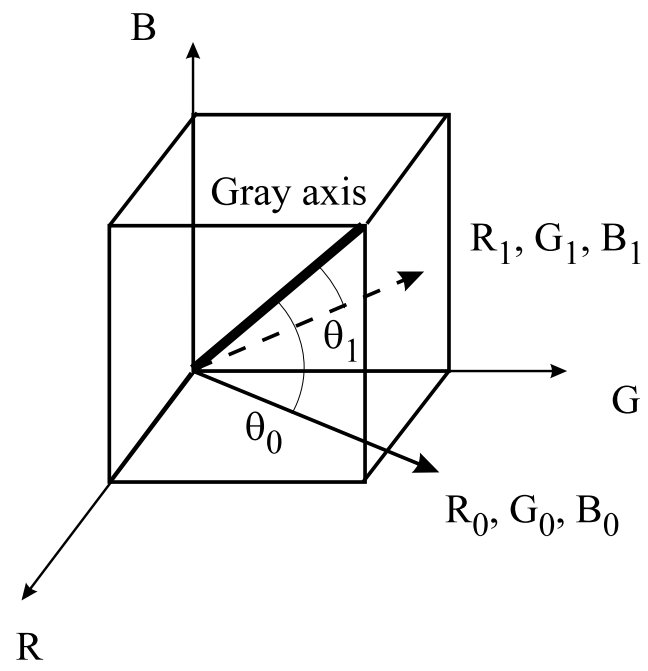

Figure 10: Shift towards gray axis

$$
B_{2}=B_{1}\left(Y / Y_{\max }\right)^{\alpha}
$$

After perceptual experiments, the best values for $\alpha$, $\beta$ and $\gamma$ were found to be: $\alpha=0.75, \beta=0.75$ and $\gamma=5$.0. Nevertheless, these values must be handled with caution, because they do not seem to be usable for images with a wide range of luminances (ie. when light sources are viewed);

- Working in the $X Y Z$ space

In ${ }^{33}$, Stone, Cowan and Beatty propose several methods to perform gamut mapping in the $X Y Z$ space. The problem they tried to solve was to print color images. However, their approach also applies to color monitors

First, they suggest to use several global transformations in such a way that the following five rules are observed in order of importance:

1. preservation of the gray axis;

2. the luminance contrast must be maximized;

3. few colors may remain outside the gamut;

4. hue and saturation shift must be minimized;

5. If saturation has to be modified, then it is better to increase it.

Three main transformations are suggested: Translation, scaling and rotation around the gray axis. Their combination may be expressed as:

$$
X_{d}=B_{d}+b s\left(W_{d}-B_{d}\right)+\operatorname{csf} R\left(X_{i}-B_{i}\right)
$$

where: $d$ stands for destination, $i$ for image, $X$ for $X Y Z$ tristimulus, $B$ for black, and $W$ for white. $R$ is the rotation matrix, $b s$ the black shift coefficient and $\operatorname{csf}$ the contrast scale factor.

A last tool, called the "umbrella transformation" can be performed. It allows to globally saturate or desaturate the set of tristimulus.

The parameters of these transformations must be interactively adjusted by an operator observing the different rules exposed before.

After the application of these transformations, the greatest part of the initial set lies in the destination gamut, except for some colors such as: Highlights, highly saturated colors, colors near black... These colors are kept outside the gamut to avoid obtaining images with too low a chromatic or achromatic contrast.

The last step is then to project these colors on the gamut boundaries. For this clipping operation, the computation of a perpendicular projection is suggested.

Currently, this mapping problem cannot be considered as completely solved. For instance, the notion of distance becomes very important, for both types of methods (clipping or global transformation). Therefore, more appropriate color spaces (such as Luv or $L a b)$ should be used.

Finally, we may notice that after this last step, color reproduction is no more objective. Due to technological constraints, visual sensations are merely suggested. For example, if the scene observer feels dazzled, then the maximum luminance of the monitor is displayed. Nothing better may be reached. .

\section{Conclusion}

In this survey, we have tried to demonstrate that color fidelity in computer graphics is a difficult and not yet completely solved problem.

It is a difficult problem because it requires knowledge in computer graphics, in signal theory (to sample computed spectra), in human vision, in technological constraints applied to color monitors...

We presented the various results known on the question today. We presented the limitations of these solutions, and some ways to future research which could improve these results.

\section{Acknowledgments}

This project has been funded in part with a grant from Région Rhône-Alpes in the framework of program Emergence. The authors would like to thank the anonymous referees for suggestions that greatly improved the paper from its original form.

\section{References}

1. Hall R., Illumination and Color in Computer Generated Images, Springer-Verlag, New-York, 1989. 
2. Beckmann P. and Spizzochino A., The scattering of Electromagnetic Waves from Rough Surfaces, Pergamon Press, New York, 1963.

3. He X. D., Torrance K.E., Sillion F.X. and Greenberg D.P., A Comprehensive Physical Model for Light Reflection, Computer Graphics, Vol. 25 (4), July 1991, 175-186.

4. Glassner A.S., Principles of Digital Image Synthesis, Morgan Kaufmann, San Francisco, 1995.

5. Schlick C., A survey of shading and reflectance models, Computer Graphics forum, Vol. 13 (2), 1994, 121-131.

6. Mc Camy C.S., Marcus H. and Davidson J.G., A color rendition Chart, Journal of Applied Photographic Engineering, Vol. 2 (3), 1976, 95-99.

7. Sillion F. and Puech C., Radiosity and Global Illumination, Morgan Kaufmann publishers, San Francisco, 1994.

8. Glassner A., An Introduction to Ray Tracing, Academic Press, 1989.

9. Wallis R., Fast Computation of Tristimulus Values by Use of Gaussian Quadrature, J. Opt. Soc. Am 65 (1), 1975, 91-94.

10. Meyer G., Wavelength Selection for Synthesic Image Generation, Computer Vision, Graphics, and Image Processing 41, 1988, 57-79.

11. Deville P. M., Merzouk S., Cazier D and Paul J.C., Spectral Data Modeling for a Lighting Application, Computer Graphics forum, Vol. 13 (3), Eurographics'94, 1994, 97-106.

12. Raso M. G. and Fournier A., A Piecewise Polynomial Approach to Shading Using Spectral Distributions, Proceedings of Graphics Interface '91, Calgary, Canada, 1991, 40-46.

13. Peercy S., Linear Color Representations for Full Spectral Rendering, Computer Graphics, Vol. 27 (3), 1993, 191-198.

14. Wandell B., The Synthesis and Analysis of Color Images, IEEE Trans on Pattern Analysis and Machine Intelligence, PAMI-91, 1987, 2-13.

15. Rougeron G., Rendu de couleurs à partir de données spectrales, Ecole des Mines de Saint Etienne, Centre Simade, Rapport de Recherche 94.7, Décembre 1994.

16. Borges C. F., Numerical determination of tristimulus values, J. Opt. Soc. Am. 11 (12), 1994, 31523161.

17. Ferwada J. A., Pattanaik S. N., Shirley P. and
Greenberg D. P., A Model of Visual Adaptation for Realistic Image Synthesis, Computer Graphics, Vol. 30 (3), 1996, 249-258.

18. Burt P.J., Attention mechanisms for Vision in a Dynamic World, ICPR 1988, 977-987.

19. Hunt R.W.G., Procedure for using a revised colour appearence model, Journal of Photographic Science, 1990, vol. 38 (4-5), 109-113.

20. Wyszecki G. and Stiles W. S., Color Science: Concept \& Methods, Quantitative Data and Formulae, Wiley-Interscience Publication, 2nd Edition, 1982

21. Kowaliski P., Vision et Mesure de la Couleur, Edition Masson, Collection Physique Fondamentale et Appliquée, 2nd Edition,1990.

22. Stevens S. S. and Stevens J. C., Brightness Function: Effect of Adaptation, J. Opt. Soc. Am 53 (3), 1963, 375-385.

23. Bodmann H.W., Haubner P. and Marsden A.M., A unified relationship between brightness and luminance, in CIE proceedings, 19th Session, Kyoto, 1979, 99-102.

24. Mac Adam D. L., Color Measurement: Theme and Variation, Springer Verlag, (Springer Series in Optical Sciences, Vol 27), 2nd Edition, 1985.

25. Tumblin J. and Rushmeier H., Tone Reproduction for Realistic Computer Generated Images, IEEE Computer Graphics \& Applications, Vol 13 (6), 1993, 42-48.

26. D'Amico D. et Taugourdeau J., Adaptation des images à la sensibilité de l'oeil, Rapport de recherche IRISA, 1993.

27. Schlick C., Quantization Techniques for Visualisation of High Dynamic Range Picture, 5th Eurographics Workshop on Rendering, Darmstad, 1994, 7-18.

28. Chiu K., Herf M., Shirley P., Swamy S., Wang C. and Zimmerman K., Spatially Non Uniform Scaling Functions for High Contrast Images, Proceedings of Graphics Interface '93, 1993, 182-191.

29. Ward G. J., A contrast-based scalefactor for luminance display, in Graphics Gems IV, P Heckbert Ed., 1994.

30. Cowan W. B., An Inexpensive Scheme for Calibration of a Colour Monitor in Terms of CIE Standard Coordinate, Computer Graphics, Vol 17 (3), 1983, 315-321.

31. Brainard D. H., Calibration of Computer Controlled Color Monitor, Color Research and Application, Vol 14 (1),1989, 23-34. 
32. Smith M., A new method of generating accurate color renderings of architectural spaces, Journal of the Illuminant Engineering Society, Winter,1993, 26-32.

33. Stone M. C., Cowan W. B. and Beatty, J. C., Color Gamut Mapping and the Printing of Digital Color Images, ACM Transactions on Graphics, Vol. 7 (4), Oct. 1988, 249-292. 